\title{
Object Tracking Using Meanshift Algorithm Combined with Kalman Filter on Robotic Fish
}

\author{
Xin Li, Wei Xiang* \\ College of Electrical \&Information Engineering \\ Southwest University for Nationalities \\ Chengdu, Sichuan, China \\ *corresponding author.Email:wei.xiang@foxmail.com
}

\begin{abstract}
This paper investigates and proposes an improved Meanshift algorithm combined with Kalman Filter aiming at the shortcomings of the Meanshift algorithm theory as well as obvious limitations of a target tracking for the independent visual robotic fish being affected by the fluctuation of the water wave. First, this new algorithm makes use of Kalman filter to obtain the initial position of the Meanshift algorithm. Then, adjust the bandwidth of the kernel function adaptively in the Meanshift tracking algorithm and use the Meanshift algorithm to obtain the position of the tracking target. Finally, we conduct a real-time tracing experiment on the independent visual robotic fish tracking a moving ball. Experimental results show that: compared with the traditional Meanshift algorithm, the improved algorithm tracks the target more accurately and the trajectory of the tracking target is more continuous. Furthermore, it reduces the number of iterations, make the algorithm run faster and improve the real - time in tracking.
\end{abstract}

Keywords- meanshift algorithm; kalman filter; kernel function; target tracking.

\section{INTRODUCTION}

In recent years, Target tracking requires effective tracking of moving objects in complex environment and becomes a hot issue in the field of computer vision research, video surveillance and target recognition applications. In the field of robotic fish research, a camera is mounted on robotic fish and it keeping on moving. In this dynamic context, tracking the moving target needs create position, speed, shape and other relevant characteristics between the consecutive image frames, so it is important to choose a good target tracking algorithm to achieve a goal of fast and accurate tracking.

The common target tracking algorithms are: adaptive Camshift algorithm based on the color histogram of the target color, which can track a single color of the target well. But when the target and background are the same color or near color, it will make the tracking window expand. Adaptive Meanshift algorithm process the video sequence, when the size of the target change, the algorithm can adaptively adjust the target area to continue tracking. The algorithm itself does not have the function of prediction, and its robustness is not strong in the case of fast moving target tracking.

In view of limitations of the algorithm mentioned above, this article put forward improved Meanshift algorithm combined with Kalman filtering algorithm, which can solved these questions above of the traditional algorithm effectively. Through the analysis of the simulation results we can know: the effect of Meanshift algorithm by wave fluctuation is obvious and the tracking effect is not ideal; while Meanshift algorithm combined with Kalman filtering algorithm of target tracking is more accurate and runs faster, improve the real-time of tracking.

\section{MEANSHIFT TRACKING ALgORITHM}

In 1975, the concept of Meanshift was first put forward by Fukunaga and Hostetlerin in an article about the probability density gradient estimation function [1]. Meanshift is a kind of target tracking way using non parametric density gradient function. It depends on calculating the density function of the feature space in the sample point rather than a priori knowledge to realize tracking. Divide a set of data into some equal number of groups according to the interval by using non parametric estimation method of statistical histogram, and the probability value of each unit is the ratio of the number of each group to total number of parameters. The principle of kernel density estimation method is similar to the histogram method, and it combines with the kernel function used to smooth the data. At present it is widely used in moving target tracking algorithm based on color histogram statistic characteristics.

\section{A. Basic theory of meanshift}

In the space of $\mathrm{D}$ dimensions, $\mathrm{S}$ represents a group of data. $k_{H}(x)$ is a multidimensional kernel function and $x$ represents a point in this space. Then, we can get an estimate density function in this space is:

$$
f(x)=\frac{1}{n} \sum_{i=1}^{n} K_{H}\left(x-x_{i}\right)
$$

Here, multidimensional kernel function $k_{H}$ is:

$$
K_{H}=\left.H\right|^{-\frac{1}{2}} K\left(|H|^{-\frac{1}{2}} X\right)
$$

In Eq.2,H is called a bandwidth matrix $d \times d$, the 
proportion of unit matrix form generally is :

$$
H=h^{2} I
$$

By taking gradient for Eq.1, we can derive:

$$
\begin{gathered}
f_{h, K}(x)=\nabla f_{h, K}(x)=\nabla\left(\frac{c_{k, d}}{n h^{d}} \sum_{i=1}^{n} k^{\prime}\left(|| \frac{x-x_{i}}{h}||^{2}\right)\right) \\
=\frac{c_{k, d}}{2 n h^{d+2}} \sum_{i=1}^{n}\left(x-x_{i}\right) k^{\prime}\left(\left.|| \frac{x-x_{i}}{h}\right|^{2}\right)
\end{gathered}
$$

$K^{\prime}(x)$ can be replaced by $g(x)$,then Eq.2 can be written as :

$$
\left[\sum_{i=1}^{n} g\left(\left.|| \frac{x-x_{i}}{h}\right|^{2}\right)\right]\left[\frac{\sum_{i=1}^{n} x_{i} g\left(\left.|| \frac{x-x_{i}}{h}\right|^{2}\right)}{\sum_{i=1}^{n} g\left(\left.|| \frac{x-x_{i}}{h}\right|^{2}\right)}-x\right]
$$

From Eq.4, we can get the first term (Eq.5), it is the kernel density estimation based on kernel function; second term is Eq.6, which is Meanshift vector that we need.

$$
\begin{aligned}
& f_{h, G}(x)=\frac{c_{g, d}}{n h^{d+2}}\left[\sum_{i=1}^{n} g\left(|| \frac{x-x_{i}}{h}||^{2}\right)\right] \\
& M_{h, G}(x)=\left[\frac{\sum_{i=1}^{n} x_{i} g\left(\left.|| \frac{x-x_{i}}{h}\right|^{2}\right)}{\sum_{i=1}^{n} g\left(|| \frac{x-x_{i}}{h}||^{2}\right)}-x\right]
\end{aligned}
$$

Basic process of Meanshift algorithm is as follows: First, by data sample points, which are a small bounded neighborhood of $X$, we can figure out Meanshift vector $M_{h . G}(x)$, Then, making a center point $x$ offset to next point $x_{c}$, if $\left\|x_{c}-x\right\|<\varepsilon \quad$ ( $\varepsilon$ is a given threshold value), the iteration process will stop. Otherwise, repeating above steps based on current center $X$, it is continuous until an end of algorithm. When the algorithm stops, it will converge to a data point and the gradient is zero, this data point is a probability density's maximum value point [2].

\section{B. The idea of the kernel function.}

Kernel-bandwidth in the Mean shift algorithm not only determines the iteration number of samples, but also reflects the size of the tracking window. Fixed kernel -bandwidth will cause the iteration number of the Mean shift algorithm increase or loss of tracking, so we propose the adaptive kernel bandwidth. First of all, let me introduce the idea of the kernel function.

Definition: X represents a D dimensional Euclidean space, $X$ is a point in this space, represented by a column vector. The norm of $x$ is $\|x\|^{2}=x^{T} x$. R represents a field of real numbers. If we have a function $K: X \rightarrow R$,.we would have a profile function $\mathrm{k}:[0, \infty] \rightarrow \mathrm{R}$. That is $K(x)=$ $k\left(\|x\|^{2}\right)$. And meet:

(1) $\mathrm{K}$ is non-negative.
(2) $\mathrm{K}$ is non-increase,namely if $\mathrm{a}<\mathrm{b}$, then $K(\mathrm{a}) \geq K(b)$

(3) $\mathrm{K}$ is piecewise continuous, and $\int_{0}^{\infty} \mathrm{k}(\mathrm{r}) \mathrm{dr}<\infty$

Then, $K(x)$ is called Kernel function.

The Meanshift algorithm calculates eigenvalue probability of the target area and candidate region respectively in the range of kernel window. According to matching the possible position of object between two adjacent frames, we can filter false targets and obtain the reliable position of targets.

We usually use the color information of object as the expression model of tracking target in practical tracking algorithm. According to the algorithm description of Mean Shift algorithm above, for a given target model, The target position in the current frame position can be obtained by comparing the length of the distance between previous position and estimation neighbourhood. When the target scale changes over time, we can use the bandwidth of kernel function repositioning to adapt to the change of target scale[3].

\section{The basic procedure of the algorithm}

(1) Initialized to all the data, and intercept the matrix of tracking target 'rect'.

(2) Calculate the histogram of tracking target 'hist1' under the weighted kernel function.

(3) Read a frame in video sequences, then randomly pick up a piece of rectangle as the same size of 'rect' to calculate the weighted histogram 'hist2'.

(4) Calculate the proportion function, if the gap of them is too large, then update new matrix center Y. Stop the iterations until a certain condition.

The specific process is shown in Fig.1.

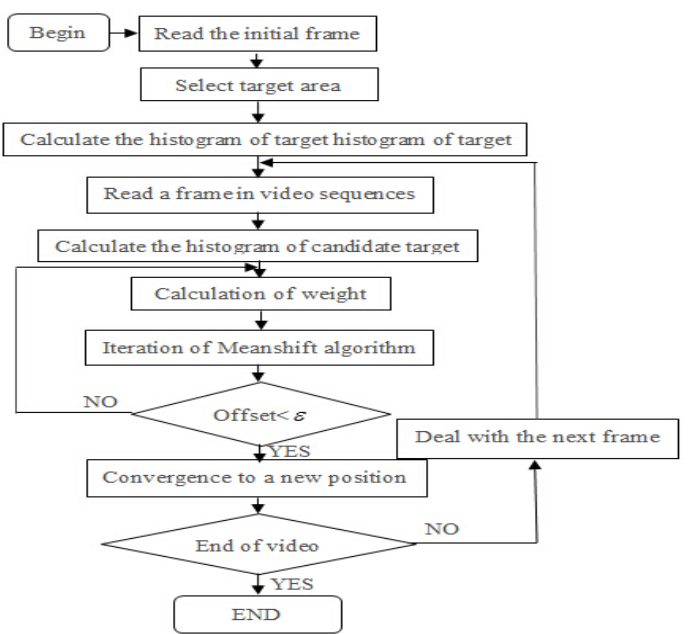

Figure 1. Detailed block diagram of traditional Meanshift algorithm.

The Mean shift algorithm based on the color histogram can be not affected by the change of target shape and proportion, so it is widely applicated in the field of video tracking. The improved adaptive Meanshift algorithm has some excellent properties in the target tracking. The algorithm with good real-time performance and single parameter can be integrated with other algorithms as a 
function module. It is not sensitive to the occlusion of target edge, deformation of target and the change of background by using the histogram of normalized kernel function as model.

\section{KALMAN FILTER}

Kalman filter is the most famous algorithm of tools for stochastic prediction estimation. In 1960, Kalman published his famous paper. In this paper, he solved the problem of discrete data linear filtering using recursive method [5].Kalman filter was named after Rudolph E.Kalman. Because of its simplicity and robustness, the Kalman filter to get the wide attention from researchers. Kalman filter assumes the posterior probability density is Gauss distribution at any time, so all the parameters of Kalman can be expressed by mean and variance. General Kalman filter includes two models, of which the signal model and observation model are:

$$
\begin{array}{r}
X(k)=M(k-1) X(k-1)+N(k) W(k) \\
Y(k)=C(k) X(k)+V(k)
\end{array}
$$

where

variables $x(k), \mathrm{y}(k), M(k), N(k), C(k), w(k)$ and $v(k)$ correspond to state vector, observation vector, state transfer matrix, input matrix, observation matrix, signal noise and observation noise of moving object respectively [6]. According to the modeling theory of Kalman filter algorithm in target tracking system proposed by Chen Dajian et[7]. We can get the system state transfer matrix $M(k)$

and observation matrix $C(k)$ are respectively:

$$
\begin{aligned}
& M(k)=\left[\begin{array}{llll}
1 & 0 & t & 0 \\
0 & 1 & 0 & t \\
0 & 0 & 1 & 0 \\
0 & 0 & 0 & 1
\end{array}\right] \\
& C(k)=\left[\begin{array}{llll}
1 & 0 & 0 & 0 \\
0 & 1 & 0 & 0
\end{array}\right]
\end{aligned}
$$

Set an initial value $t=1, \sigma_{\omega}=\sigma_{v}=5, \sigma_{\omega}$ and $\sigma_{v}$ are obtained according to experience. The state vector at the initial time is $\mathrm{x}_{0}=\left[\begin{array}{llll}\mathrm{x}_{0} \mathrm{y}_{0} & 0 & 0\end{array}\right]^{\mathrm{T}}, \mathrm{x}_{0}$ and $\mathrm{y}_{0}$ are position coordinate of target for the initial frame. Then, we establish Kalman filter model of moving target.

Proposal and implementation of Kalman filter theory, not only overcomes the limitations of the Wiener filter theory, but also has been widely used in engineering, especially in the communications, navigation, guidance, control and other modern engineering.

\section{MEANSHIFT ALGORITHM COMBINED WITH KALMAN FILTER}

The traditional Meanshift algorithm uses color histogram as the representation model and gets Taylor expansion from the starting points of the target, namely finding the target in the starting position. Tracking performance of the algorithm will be affected when the target is moving fast. These disadvantages of Mean Shift algorithm often blames that the starting position in the frame is not ideal. If we can find an effective method of predicting the motion model to predict the position of the object in the current frame by the modeling of target motion in the past every moment, we can solve the relevant problems of Mean Shift algorithm in practical application.

We can solve the problems existed in the Meanshift algorithm well by using filter algorithm. First, filter the state space of target. Then, the Mean shift algorithm tracks by iteration in the estimated position. In view of the target tracking system and the requirements of the real-time, the paper proposes an improved Meanshift algorithm combined with Kalman Filter to realize real-time tracking and position of the fast moving targets.

a) Determine the initial state vector of Kalman filter and initialize the other necessary parameters and use the core position of tracking frame set manually as the initial position $\mathrm{X} 0$.

b) Using Kalman filter after initialization predicts the current candidate target position of the current frame according to the previous state value $\mathrm{X} 0$.

c) Taking the candidate target position based on Kalman filter prediction as Meanshift algorithm's iterative initial point, start Meanshift iterative process until convergence to get the true target position.

d) Next, make the true target position gained by Meanshift algorithm as the observation vector to update the Kalman filter

e) Using the updated Kalman filter according to the same principle, repeat from step (2) to step (4) and figure out the target location of the next frame[2].

The specific process is shown in Fig.2.:

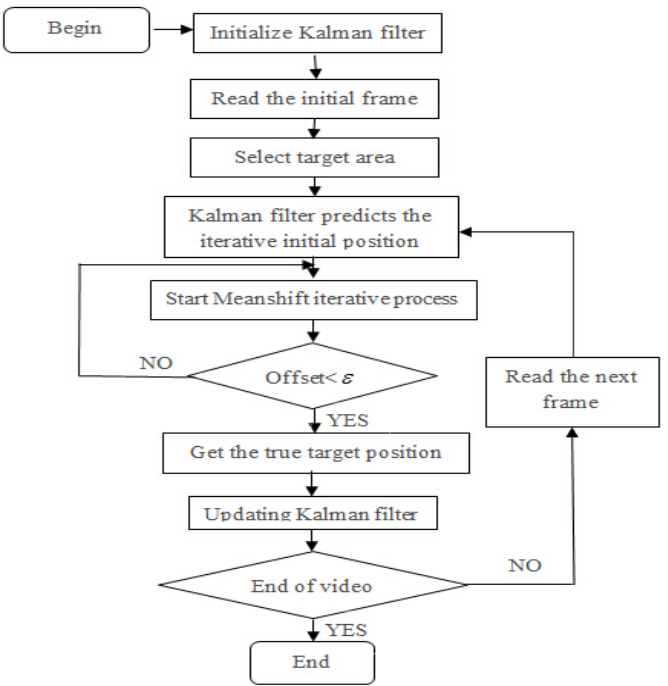

Figure 2. Detailed block diagram of improved Meanshift algorithm. 


\section{V.THE EXPERIMENT RESULTS AND DATA ANALYSIS OF MATLAB SIMULATION}

In order to verify rapidity and accuracy of Meanshift algorithm combined with Kalman Filter in real-time target tracking, and visually reflect the superiority of the improved algorithm compared with the traditional one, we conduct the simulation experiment of real-time tracking in MATLAB on the independent visual robotic fish tracking a moving ball.

First, we record a video that a ball move from slow to fast in the water, and extract frame sequence of the video. During the experiment we mapping the images from RGB color space to feature space and quantize them to $16 \times 16 \times$ 16 feature level. Then, assume the ball in the initial frame as the target to track. Finally, we respectively use the traditional algorithm and improved algorithm to carry on the real-time target tracking in the MATLAB observing the situation of tracking. The analysis of experimental results is as follows:

\section{A. MATLAB simulation of continuous path.}

Through the simulation experiment, we draw trajectory of the target(the ball) tracking in sequence of video frames by using two kinds of algorithm respectively in MATLAB. Tracking results partly are shown in Fig.3 and Fig.4:

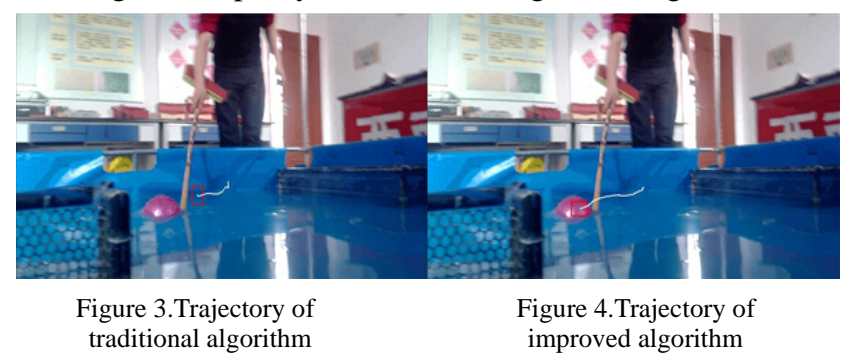

By analyzing above results in Fig.3, we can find: When the ball moves with a smaller velocity, trajectory of tracking by using the traditional Meanshift algorithm is continuous with relative accuracy. But when the ball has a rather fast velocity, it begins to lose frames, so that the final failure of tracking, and trajectory is not continuous. From Fig.4 we can see: When the velocity of the ball increases, we can get the position of the target predicted by Kalman filter. The tracking trajectory is continuous without losing frames. And using Meanshift algorithm combined with Kalman Filter can track the target accurately.
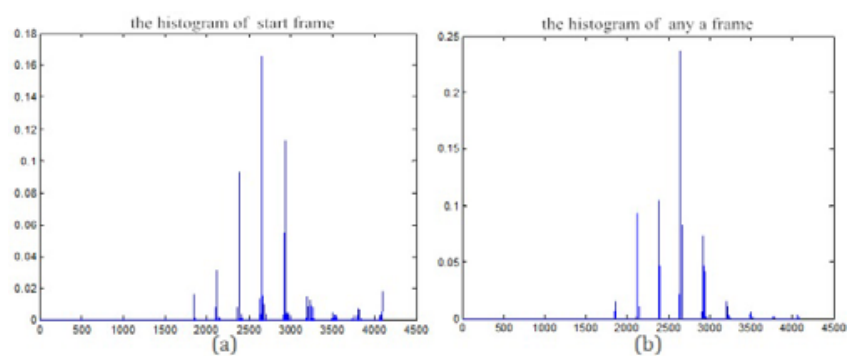

Figure 5. Histogram of start frame and any a frame
We select the histogram of target in start frame and the histogram of target in any a frame. As shown in Fig.5, the matching degree is higher illustrating adaptive scale of tracking is good and it is not easy to lost frames.

B. The analysis of validity :

- The accuracy of the tracking:

In the analysis of $\mathrm{A}$, we can know the traditional Meanshift algorithm may have the situation that it can't identify accurately due to the change of velocity. We contrast experiments repeatedly by using the two kinds of algorithms to track 180 frame images, and show the results in Fig.6 as example.
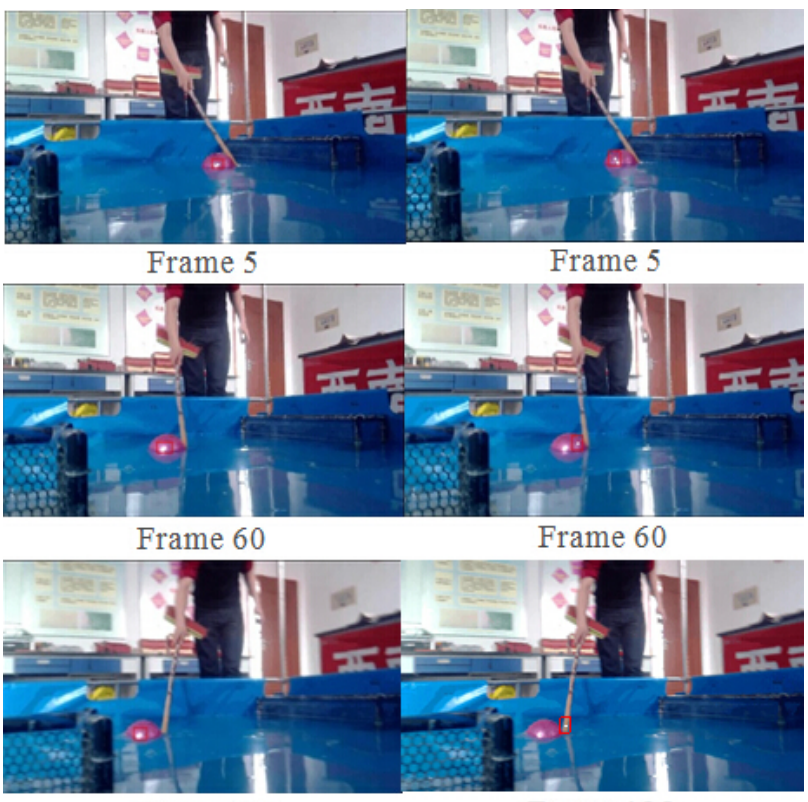

Frame 125

Frame 125

Figure 6. Results of MATLAB simulation

Throug comparing we can find: In the fifth frame, the tracking effect of the two kinds of algorithms is similar when the target is in the low speed. In the sixtieth frame, the velocity of the target begins to increase, then you can see the traditional algorithm has not accurate identification. Recognition box of tracking begins not to follow the target But the improved algorithm can accurately identify. In the 125th frame, the traditional algorithm has lost frames and the recognition box of tracking can not to follow the target completely while the improved algorithm still can. Thus, it can directly reflect the accuracy and adaptability of the Meanshift algorithm combined with Kalman Filter is better than the traditional Meanshift algorithm.

- The velocity of tracking

In order to compare the performance of two kinds of algorithm in tracking speed, we calculate the run - time of algorithm respectively. The experimental results show: The run - time of the traditional Meanshift algorithm is 41.5586s and the run - time of the Meanshift algorithm combined with Kalman filter is 32.6691s.Comparison shows: the improved algorithm mentioned in the article increases the 
run time due to adding the Kalman filter, But the Kalman filter reduces the number of the iterations of the traditional algorithm making the computing time is reduced on the whole. Therefore, Tracking speed of the proposed algorithm in paper is superior.

\section{SUMMARY}

In the actual tracking, the traditional algorithm using color histogram as the representation model just focus on the color features of the target itself, but completely loses the information of the state space and motion. When there are large external disturbances, a fast moving target or sudden changes in light intensity, it often leads to the failure of tracking algorithm. This paper proposes an improved Meanshift algorithm combined with Kalman filter aiming at the shortcomings of the Meanshift algorithm theory. Through establishing Kalman filter model of moving target in the scene, according to the trend of target motion, we can estimate the possible target position of the next frame. Then, we use the Mean Shift algorithm in the kernel window range to search the optimal matching with the position as the starting point. Experiments show that: Especially when it meets the constraint conditions of Kalman filter, the accuracy and adaptability of the Meanshift algorithm combined with Kalman Filter is better than the traditional Meanshift algorithm.

\section{ACKNOWLEDGEMENTS}

This work was financially supported by Innovative Research Team of the department of Sichuan Province (15TD0050) and the Fundamental Research Funds for Central University, Southwest University for Nationalities (13NZYQN10).

\section{REFERENCES}

[1] Fukunaga K.and L.D. Hostetler. The estimation of the gradient of a density function with application in pattern recognition. IEEE Trans. Information Theory. 1975, 21(1):30-42.

[2] Yang Jianwei. Research on object tracking using Meanshift combined with Kalman filtering.Harbin Institute of Technology,2010.

[3] Comaniciu D,Rarnesh V and Meer P. Kernel-based Object Tracking. IEEE.Trans. Pattern Analysis and Machine Intelligence,2003,v01. 25,PP. 564.577.

[4] Gu Fengqiang algorithm on Mean Shift video tracking based on. the Xi'an Electronic and Science University. 2009

[5] Kalman R.E.A New Approach to Linear Filtering and Prediction Problems.Transaction of the ASME-Journal of Basic Engineering, 1960, 35-45..

[6] WangShuai,Zhao Hui,Zhou Linchuan. An Algorithm of Object Tracking Based on Meanshift. Shandong University.China.

[7] Chen Dajian . Li Feixiang. Zhang Zihao. Calman. Fu Xin filter algorithm in target tracking system modeling using [J]. optoelectronic technology application of.2006.06.21 69-72 